\title{
Zidovudine-Poly(L-Lactic Acid) Solid Dispersions with Improved Intestinal Permeability Prepared by Supercritical Antisolvent Process
}

\author{
VALQUÍRIA M. H. YOSHIDA, ${ }^{1,2}$ VICTOR M. BALCÃO, ${ }^{1,3}$ MARTA M. D. C. VILA, ${ }^{1}$ JOSÉ M. OLIVEIRA JÚNIOR, ${ }^{4}$ \\ NORBERTO ARANHA, ${ }^{4}$ MARCO V. CHAUD, ${ }^{1}$ MARIA P. D. GREMIÃO ${ }^{2}$ \\ ${ }^{1}$ Laboratory of Biomaterials and Nanotechnology of the University of Sorocaba, University of Sorocaba, Sorocaba, São Paulo, Brazil \\ ${ }^{2}$ School of Pharmaceutical Sciences, São Paulo State University-UNESP, Araraquara, São Paulo, Brazil \\ ${ }^{3}$ Centre of Biological Engineering, University of Minho, Braga, Portugal \\ ${ }^{4}$ Laboratory of Applied Nuclear Physics, University of Sorocaba, Sorocaba, São Paulo, Brazil
}

Received 22 September 2014; revised 13 January 2015; accepted 14 January 2015

Published online 10 February 2015 in Wiley Online Library (wileyonlinelibrary.com). DOI 10.1002/jps.24377

\begin{abstract}
A supercritical antisolvent (SAS) process for obtaining zidovudine-poly(L-lactic acid) (PLLA) solid dispersions (SDs) was used to attain a better intestinal permeation of this drug. A $3^{2}$ factorial design was used, having as independent variables the ratio $3^{\prime}$-azido-2'3'dideoxythymidine (AZT)-PLLA and temperature/pressure conditions, as dependent variables the process yield and particle macroscopic morphology. AZT-PLLA production batches were carried out by the SAS process, and the resulting products evaluated via scanning electron microscope, X-ray diffraction, differential scanning calorimetry, and Fourier transform infrared analyses. From the nine possible combinations of tests performed experimentally, only one combination did not produced a solid. The L3 batch of SD, produced with 1:2 (AZT-PLLA) ratio, resulted in a 91.54\% yield, with 40\% AZT content. Intestinal permeability studies using the AZT-PLLA from L3 batch led to an AZT permeability of approximately $9.87 \%$, which was higher than that of pure AZT ( $3.84 \%)$. AZT remained in crystalline form, whereas PLLA remained in semicrystalline form. AZT release is controlled by a diffusion mechanism. It has been demonstrated that it is possible to use PLLA carrier and SAS process to obtain SD, in a single step. (C) 2015 Wiley Periodicals, Inc. and the American Pharmacists Association J Pharm Sci 104:1691-1700, 2015
\end{abstract}

Keywords: supercritical antisolvent process; supercritical fluids; zidovudine; poly(L-lactic acid); solid dispersion; oral absorption; gastrointestinal transit; everted rat intestinal sacs; permeability

\section{INTRODUCTION}

The treatment of Acquired Immune Deficiency Syndrome (AIDS) is a public health problem throughout the world. Conventional dosage forms have contributed to improve both the quality of life and life expectancy of patients infected by the Human Immunodeficiency Virus, ${ }^{1}$ but numerous factors still hinder the best use of the available therapeutic arsenal. ${ }^{2,3}$ The zidovudine (3'-azido-2' $3^{\prime}$-dideoxythymidine; AZT) antiretroviral activity was first observed by Mitsuya et al., ${ }^{4}$ being licensed in the United States of America in 1986 for the treatment of advanced HIV infection. In 1987, the use of AZT was expanded in an open clinical study involving patients with AIDS and/or those with HIV infection at high risk for developing AIDS; however, this clinical study received a notice of compliance in $1990 .^{5}$ In the Biopharmaceutics Classification System, ${ }^{6}$ AZT (clog $P$ 0.043999 and solubility of $10 \mathrm{mg} / \mathrm{mL}, \mathrm{pKa} 9.8$ ) is in the lowpermeability and high-solubility drugs group (class III).

The poly(L-lactic acid) (PLLA) is an aliphatic polyester that degrades into L-lactic acid, a natural metabolic product. PLLA is a semicrystalline aliphatic polyester with a melting point in the range of $175^{\circ} \mathrm{C}-185^{\circ} \mathrm{C}$, glass transition temperature in the range of $60^{\circ} \mathrm{C}-65^{\circ} \mathrm{C}$ and molecular weight in the range of

Correspondence to: Marco Vinicius Chaud (Telephone: +55-15-33162260; Fax: +55-15-33162260; E-mail: marco.chaud@prof.uniso.br)

Journal of Pharmaceutical Sciences, Vol. 104, 1691-1700 (2015)

(C) 2015 Wiley Periodicals, Inc. and the American Pharmacists Association
5000-700,000 Da. ${ }^{7,8}$ Aliphatic polyesters are used as biocompatible and biodegradable polymers for implantable controlledrelease systems (including rods, cylinders, tubes, films, fibers, beads, and balls) and injection systems (including microcapsules, microspheres, and nanoparticles) for drugs of human and veterinary use. The rate of biodegradation and drug release characteristics from these controlled-release systems made of aliphatic polyesters can be controlled by altering the physicochemical properties of the polymers, such as crystallinity, hydrophobicity, monomer stereochemistry, copolymer ratio, and polymer molecular weight. ${ }^{7,8}$

Among controlled drug delivery systems, one can find systems such as polymeric solid dispersions (SDs), nanoparticles, solid lipid nanoparticles, liposomes, nanoemulsions, dendrimers, conjugated drugs, and hollow microspheres.

The established generation systems, either in micro- or nanoscale, using conventional methodologies such as coprecipitation, coacervation, and solvent evaporation polymerization, use large amounts of organic solvents and/or surfactants that in general are harmful to the environment. ${ }^{9,10}$ The aforementioned methods used to produce particles commonly exhibit low rates of drug entrapment and difficulties in scaling, and most often require further treatment for particle removal from the solvent and/or impurities. Although the pharmaceutical industry shows interest in innovative technologies involving production of microparticles and nanoparticles, disposal of solvents strongly limits the industrial production and 
generates increased costs. ${ }^{11}$ Hence, continued efforts to develop other technologies that minimize the problems related to pollution, toxicity, and ease of production scale-up has been the subject of several studies. ${ }^{12-15}$

In the processes to achieve these goals, the supercritical fluid (SCF) technology occupies a prominent role. The major advantages of the SCF process are the simplicity of production steps, the low temperature used in the process, and the use of $\mathrm{CO}_{2} \cdot{ }^{16}$ The supercritical antisolvent (SAS) process is based on the decrease of solvent power of a polar organic solvent, in which the substrate of interest (a pharmaceutical-active ingredient and/or polymer) is dissolved. ${ }^{17}$ Subsequently, this saturated solution undergoes a SCF process to cause precipitation and recrystallization of the substrate of interest. Hence, the SCF is used to extract the solvent from this saturated solution. ${ }^{11,15}$ The SAS process is easily scaled-up and has been widely studied because of its potential in the production of microparticles and nanoparticles with controlled particles sizes and morphology. ${ }^{18-20}$ The SAS process is usually carried out at temperatures ranging from $35^{\circ} \mathrm{C}$ to $60^{\circ} \mathrm{C}$, and is particularly useful when thermolabile compounds need to be micronized. ${ }^{21}$

Sekiguchi and $\mathrm{Obi}^{22}$ first introduced the term SD, which refers to the dispersion of one or more active ingredients in an inert carrier in a solid state, frequently prepared by the melting method, solvent method, or melting solvent method. SDs are often used to increase the equilibrium solubility of poorly soluble drugs in water, ${ }^{23,24}$ and SDs prepared with poorly soluble carriers have been used to control the dissolution rate of watersoluble drugs, with the aim of obtaining controlled-release systems. The PLLA is a copolymer semicrystalline, slightly soluble in water and used in modified drug delivery systems.

It has been demonstrated in this research effort that it is possible to use PLLA as a carrier for preparing AZT SD to control the drug dissolution rate and SAS process to obtain SD, so as to attain a better intestinal absorption of the AZT.

\section{MATERIAL AND METHODS}

\section{Chemicals}

3'-Azido-2'3'-dideoxythymidine was kindly provided by Cristalia (lot 0035/13, purity grade 99.849\%; Itapira, São Paulo, Brazil). PLLA was manufactured by the Biomaterials Research Group of the Faculty of Medical and Health Sciences at PUC-SP and kindly provided by Prof. Eliana Aparecida de Rezende Duek. The TC199 tissue culture medium was composed of $8.47 \mathrm{~g} \mathrm{NaCl}, 0.34 \mathrm{~g} \mathrm{KCl}, 0.126 \mathrm{~g} \mathrm{CaCl}_{2} \cdot 2 \mathrm{H}_{2} \mathrm{O}$, $0.595 \mathrm{~g} \mathrm{NaHPO}_{4}$, and $0.018 \mathrm{~g}$ glucose, to prepare $1 \mathrm{~L}$ of solution with $\mathrm{pH}$ 7.4. All other chemicals used were of reagent or pharmaceutical grade.

\section{Analytical and Processing Equipment}

The SCF system utilized for this research work was from Waters Corporation (SAS-RESS Combined System, Pittsburgh, Pennsylvania). For dissolution and the removal of the dispersed gas in solution was used bath ultrasound (Unique USC2800A; Indaiatuba, Brazil). All spectrophotometric readings were carried out in a UV-Vis spectrophotometer from Shimadzu (MultiSpec-1501; Kyoto, Japan). All dissolution tests were carried out using a dissolution apparatus from American Lab (AL1000; Charqueada, Brazil). Scanning electron microscopy analyses were carried out in a scanning electron microscope (SEM) from Leica (Leo 440i; Cambridge, England), following gold sputter coating of the particles with a SputterCoating plating device also from Leica (SC7620). All infrared spectrophotometric analyses were performed in a Fourier transform infrared (FTIR) spectrophotometer from Thermo Scientific (Nicolet 6700; Madison, Wisconsin) with Fourier transform, coupled with an Omni Smart-Sampler also from Thermo Scientific. All differential scanning calorimetry (DSC) analyses were carried out in a Differential Scanning Calorimeter from Shimadzu (DSC-60), equipped with an accessory flow-controller (60A) and an interface for data acquisition and control (TA60WS) also from Shimadzu. X-ray powder diffraction analyses were carried out in X-ray diffractometer from Philips Analytical (X'Pert-MPD; Almelo, The Netherlands).

\section{EXPERIMENTAL PROCEDURES}

\section{Experimental Design}

To reduce the number of runs needed to obtain the highest amount of information on product performance, the screening was planned using an experimental (factorial) design. Specifically, a randomized plan was employed, as two factors were evaluated each at three levels, and experimental trials were performed with all nine possible combinations. A $3^{2}$ factorial design was thus used, producing nine runs. In the present research effort, the ratio of AZT-PLLA $\left(X_{1}\right)$ and the conditions of temperature and pressure $\left(X_{2}\right)$ were selected as independent variables. Mixtures of AZT-PLLA were evaluated at ratios of $2: 1,1: 1$, and $1: 2$, whereas the temperature/pressure of the process was evaluated at $45^{\circ} \mathrm{C} / 85$ bar, $45^{\circ} \mathrm{C} / 100$ bar, and $45^{\circ} \mathrm{C} / 135$ bar.

\section{Preparation of Production Batches}

The AZT-PLLA solution was prepared at $15 \%(\mathrm{w} / \mathrm{v})$ by dissolving of AZT in $5 \mathrm{~mL}$ of ethanol and PLLA in $95 \mathrm{~mL}$ of dichloromethane, and then the solution of AZT was mixed with the solution of PLLA. A bath with ultrasound (frequency $40 \mathrm{kHz}, 30^{\circ} \mathrm{C} / 30 \mathrm{~min}$ ) was used to remove any gases and homogenize the AZT-PLLA solutions. The schematic representation of the SAS process is depicted in Figure 1. During the production trials, the temperature was maintained at $45^{\circ} \mathrm{C}$ and the pressure of the system was varied at 85, 100, and 135 bar. When steady-state conditions were reached in the high-pressure vessel (V1; see Fig. 1), the AZT-PLLA solution was released into V1 chamber by a high-pressure pump (P2; see Fig. 1) at a flow rate of $0.75 \mathrm{~mL} / \mathrm{min}$ and further sprayed through a stainless steel capillary tube $(101.6 \mu \mathrm{m}$ internal diameter $)$. The spraying was maintained for $27 \mathrm{~min}$, followed by another $20 \mathrm{~min}$ of $\mathrm{CO}_{2}$ spraying supplied at a rate of $3.0 \mathrm{~mL} / \mathrm{min}$ to remove any residual organic solvent. When the cleaning step was completed, the high-pressure vessel (V1) was slowly depressurized and product samples were collected for full physicochemical characterization.

\section{AZT Loading and AZT Loading Efficiency}

To determine the free drug mass or just adsorbed to the particle surface, an analytically weighed mass of the samples (L3, L5, and L9) was dispersed in $50 \mathrm{~mL}$ of purified water ( $\mathrm{pH} \mathrm{7)}$ and stirred for $15 \mathrm{~min}$ in an ultrasound bath $(40 \mathrm{kHz})$ and filtered under vacuum. The concentration of AZT in the filtrate was determined by UV spectroscopy (266 nm). Samples were 


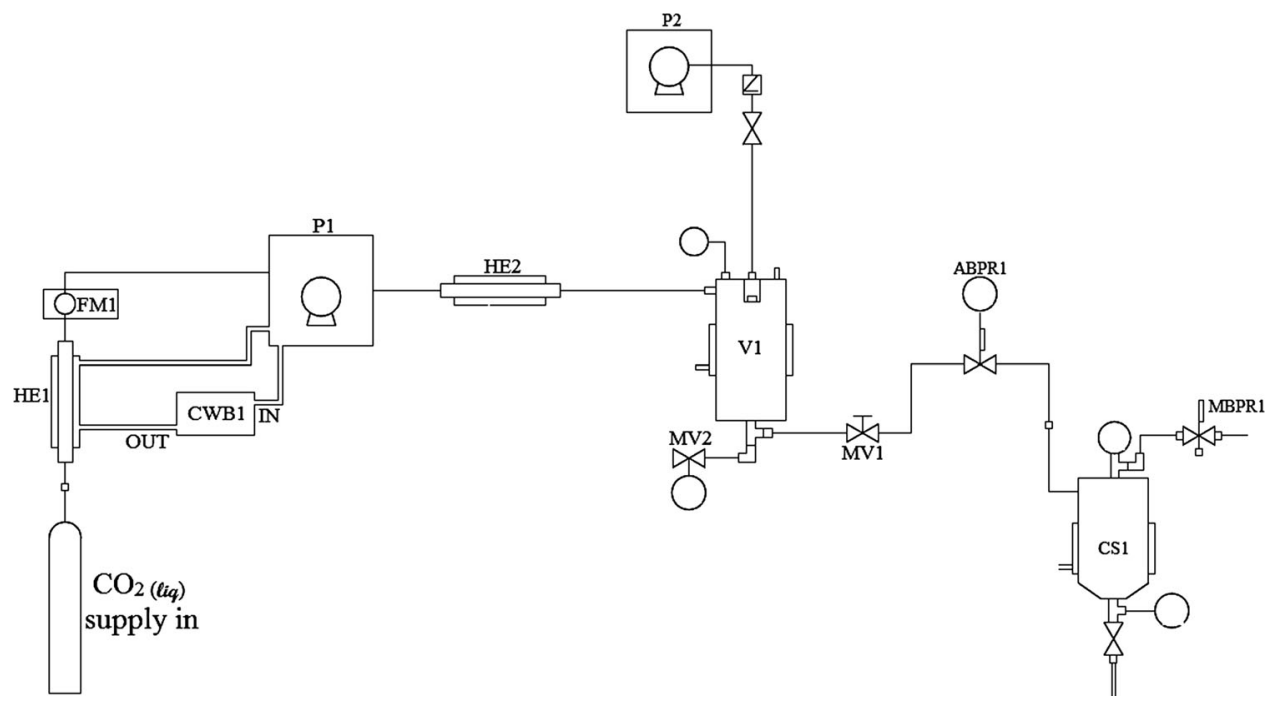

Figure 1. Schematic representation of the SAS process.

transferred to a volumetric flask and diluted with purified water ( $\mathrm{pH} \mathrm{7)}$ to $100 \mathrm{~mL}$ and maintained for $60 \mathrm{~min}$ in ultrasound bath $(40 \mathrm{kHz}), 65^{\circ} \mathrm{C}$. The dispersion was filtered and AZT concentration was determined by UV spectroscopy ( $266 \mathrm{~nm})$. The drug loading of SD was expressed as the mass fraction of encapsulated drug relative to the mass of the sample, and was calculated using the following equation:

$$
\text { Drug loading }(\%)=\frac{\text { Mass of encapsulated drug }}{\text { Total mass of particle }} \times 100
$$

The drug loading efficiency was then determined as:

$$
\begin{aligned}
\text { Drug loading efficiency }(\%)= & \frac{\operatorname{Drug} \operatorname{loading}(\%, \mathrm{w} / \mathrm{w})}{\text { Theoretical drug loading }(\%, \mathrm{w} / \mathrm{w})} \\
& \times 100
\end{aligned}
$$

\section{Dissolution Test}

The release rate profile of AZT from the product samples (30 mg of each sample was weighed analytically) was examined in vitro at predetermined time, using a USP II dissolution apparatus. The dissolution medium contained $900 \mathrm{~mL}$ of simulated gastric fluid, without pepsin, at $\mathrm{pH} 1.2$, with the temperature maintained at $37 \pm 0.5^{\circ} \mathrm{C}$. At predetermined time intervals, $5 \mathrm{~mL}$ samples were withdrawn and duly filtered. The volume of the dissolution medium was kept constant by means of immediate reposition following sample withdrawal. The test was carried out under sink conditions, and the concentration of AZT dissolved in the medium was then determined via spectrophotometric readings at $266 \mathrm{~nm}$, as described above.

\section{Kinetic Modeling of Drug Dissolution}

Three mathematical models for interpreting dissolution kinetics were fitted to the AZT release data gathered from the dissolution tests, using the software SigmaPlot ${ }^{\mathrm{TM}}$ 10.0. The equations $\ln Q_{t}=\ln Q_{0} K_{1} t, Q_{t}=Q_{0}+K_{\mathrm{H}} t^{1 / 2}$ and $Q_{t} / Q_{\infty}=K_{\mathrm{KP}} t^{n}$ were used to test the models of the first order, Higuchi, and Korsmeyer-Peppas, respectively. In these models, $Q_{t}$ is the amount of drug dissolved at any time $t, Q_{0}$ is the initial amount of drug in the solution, $K_{1}$ is a first-order release constant, $K_{\mathrm{H}}$ is a Higuchi dissolution constant trend, $K_{\mathrm{KP}}$ is a constant incorporating structural and geometric characteristic of the drug dosage form, and $n$ is a release exponent. ${ }^{25}$

\section{Scanning Electron Microscopy Analyses}

Particles were analyzed for morphological characteristics using a SEM and a Sputter Coater. Briefly, samples for SEM analysis were prepared by lightly sprinkling the powdered particles on an adhesive double-face tape kept adhered on an aluminum stub. Samples were then sputter-coated with gold to a thickness of about $300 \AA$, under inert (argon) atmosphere with highvacuum evaporator. Samples were randomly scanned, photomicrographed at $5 \mathrm{kV}$, and magnified at $50 \times$ up to $3000 \times$.

\section{Analyses via Infrared Spectrophotometry with Fourier Transform}

The infrared spectra of AZT, PLLA, and SDs were gathered in the range of wavenumber from 4000 to $675 \mathrm{~cm}^{-1}$ with a resolution of $4 \mathrm{~cm}^{-1}$, using an infrared spectrophotometer with Fourier transform. FTIR spectra were obtained in ATR mode, using the accessory Omni Smart-Sampler (Thermo Scientific).

\section{DSC Analyses}

All samples were subjected to analyses in a Differential Scanning Calorimeter, equipped with an accessory flow-controller, a software, and dedicated interface for data acquisition and control. Samples weighing approximately $2 \mathrm{mg}$ were placed inside aluminum pans, duly sealed using a crucible, and subject to a heating cycle from $30^{\circ} \mathrm{C}$ to $350^{\circ} \mathrm{C}$ at a constant heating rate of $10^{\circ} \mathrm{C} / \mathrm{min}$ under inert (nitrogen) atmosphere.

\section{X-ray Diffraction Analyses}

X-ray diffractograms of samples of AZT, PLLA, and SDs were obtained in a X-ray diffractometer using filtered $\mathrm{Ni}$ and $\mathrm{Cu}$ $\mathrm{K} \alpha$ radiation $(\mathrm{Cu} \mathrm{K} \alpha$ of $\lambda=1.54060 \AA)$. Analyses were performed at a diffraction angle of $2 \theta$ (from $4^{\circ}$ to $40^{\circ}$, step: $0.02^{\circ}$, speed $0.05 \%$ s) with voltage and current set at $40 \mathrm{kV}$ and $40 \mathrm{pA}$, respectively. 


\section{In Vitro Intestinal Permeation Studies Using Everted Rat Intestinal Sacs}

3'-Azido-2' 3 '-dideoxythymidine intestinal permeation was evaluated in vitro using the everted rat intestinal sac (ERIS) model. The ERIS was obtained from adult male Wistar rats, weighing from 250 to $300 \mathrm{~g}$ and fasted for a period of $8 \mathrm{~h}$. This period of fasting was established according to the scientific literature related to the investigation of permeability of substances using the proposed in vitro model. ${ }^{23,26,27}$ Rats were anesthetized using halothane solution and pentobarbital (intraperitoneal anesthetic $0.5 \mathrm{mg} / \mathrm{Kg}$ ) and the small intestine was immediately dissected, washed with TC199 medium previously kept at $10^{\circ} \mathrm{C}$, and submerged in $20 \mathrm{~mL}$ of oxygenated $\left(\mathrm{O}_{2}-\mathrm{CO}_{2}\right.$ ratio of 95:5) TC199 tissue culture medium at the same temperature. The intestine was then everted using a flexible rod with its end protected by a thin silk fabric (mini brush); one extremity was closed using suture thread, and the intestinal segment filled with TC199 solution. The intestinal segment $(6 \mathrm{~cm})$ was ligated at one end, and was closed at the other end. The incubation medium was added of the unprocessed AZT, AZT-PLLA SD, or AZT-PLLA physical mixture, respectively, 5, 6, and $6 \mathrm{mg}$. Incubation of the closed ERIS was carried out at $37^{\circ} \mathrm{C}$ under gentle agitation. After an incubation time of $60 \mathrm{~min}$, samples $(n=6)$ of both the inner fluid (in contact with the serous membrane of the ERIS) and outer fluid (in contact with the mucous membrane of the ERIS) were filtered and analyzed by UV-Vis spectrophotometry at a wavelength of $266 \mathrm{~nm}$. After collecting the data, values of the samples were analyzed by using unpaired Student's $t$-test, at the 0.05 level, using OriginPro 8 (OriginLab ${ }^{\circledR}$ ) software.

The protocol for the study of intestinal drug permeation using ERIS was duly approved by the Ethics Committee on Animal Use (CEUA) of the Federal University of São Carlos (UFSCAR), under No. 003/2011, catering to CFMV Res No. $1000 / 2012$.

\section{RESULTS}

\section{Selection of Processing Parameters for Preparation of SDs Under Supercritical Conditions}

Results of both yield (\%) and appearance of SDs of AZT with PLLA are displayed in Table 1. Batches of L1 through L9 correspond to the formulations obtained via the $3^{2}$ experimental factorial design. The independent variable $X_{1}$ (in three different levels, viz., $+1,0$, and -1 ) and the independent variable $X_{2}$ (also in three different levels, viz., $+1,0$, and -1 ) resulted in the production of different solid precipitate aspects, except for L1. The results obtained guided the selection of samples for further physicochemical analyses. With respect to yield and appearance of the product, the L3 SD (91.54\% yield, solid particulate with uniform appearance) was selected for both physicochemical characterization and ex vivo study of intestinal permeation. Additionally, considering the same dependent variables, SDs L5 $(59.06 \%$ yield, solid particulate with filamentous nonuniform aspect) and L9 (51.50\% yield, solid particulate with filamentous nonuniform aspect) were also selected. Formulation L3 corresponds to a ratio of AZT-PLLA of 1:2 (level +1$)$ and temperature of $45^{\circ} \mathrm{C}$ and pressure of 85 bar (level -1 ). This formulation displayed a high degree of uniformity and was free of lumps. The other formulations selected (L5 and L9) exhibited a varying degree of uniformity and presented large lumps.
Table 1. Experimental Results of $3^{2}$ Factorial Design

\begin{tabular}{|c|c|c|c|c|}
\hline \multirow[b]{2}{*}{$\begin{array}{l}\text { Batch } \\
\text { Code }\end{array}$} & \multicolumn{2}{|c|}{ Coded Values } & \multirow[b]{2}{*}{$\begin{array}{l}\text { Yield } \\
(\%)\end{array}$} & \multirow[b]{2}{*}{$\begin{array}{l}\text { Appearance } \\
\text { of Product }\end{array}$} \\
\hline & $\begin{array}{c}X_{1} \\
\text { (AZT-PLLA) }\end{array}$ & $\begin{array}{c}X_{2} \\
(T, P)\end{array}$ & & \\
\hline L1 & +1 & +1 & 0 & - \\
\hline L2 & +1 & 0 & 51.50 & +++ \\
\hline L3 & +1 & -1 & 91.54 & + \\
\hline L4 & 0 & +1 & 78.74 & +++ \\
\hline L5 & 0 & 0 & 59.06 & ++ \\
\hline L6 & 0 & -1 & 75.80 & +++ \\
\hline $\mathrm{L} 7$ & -1 & +1 & 77.29 & +++ \\
\hline L8 & -1 & 0 & 62.50 & +++ \\
\hline L9 & -1 & -1 & 51.50 & ++ \\
\hline Coded values & $X_{1}$ & $X_{2}$ & & \\
\hline-1 & $2: 1$ & $45^{\circ} \mathrm{C}, 85 \mathrm{bar}$ & & \\
\hline 0 & $1: 1$ & $45^{\circ} \mathrm{C}, 100 \mathrm{bar}$ & & \\
\hline+1 & $1: 2$ & $45^{\circ} \mathrm{C}, 135 \mathrm{bar}$ & & \\
\hline
\end{tabular}

-, Product not obtained; +, solid particles of uniform aspect; ++ , solid particulate of filamentous and nonuniform aspect; +++ , nonparticulate solids.

\section{Scanning Electron Microscopy Analyses}

Results from SEM analyses can be found in Figure 2. The micrographs of AZT and PLLA unprocessed with magnification of $400 \times$ (Figs. $2 \mathrm{a}$ and $2 \mathrm{~b}$ ) show the crystalline form of the drug ${ }^{28,29}$ and polymer filamentous form of the polymer. Figures $2 \mathrm{c}$ and 2d (L3) show significant reduction in size and diameter of the PLLA fibers and size of the AZT crystals. Both the L5 (Fig. 2e) and L9 (Fig. 2f) formulations exhibited a disorganized morphology when compared with L3.

\section{DSC Analyses}

Results from the DSC analyses of pure AZT, PLLA, and SD formulations (viz., L3, L5, and L9) are displayed in Figure 3. As can be observed from a close inspection of Figure 3, the thermogram of pure AZT exhibited an endothermic peak in the temperature range between $120^{\circ} \mathrm{C}$ and $130^{\circ} \mathrm{C}$, corresponding to the melting point of AZT crystals $\left(T_{\text {onset }}=123^{\circ} \mathrm{C}\right.$, with an enthalpy of absorption of $100.6 \mathrm{~J} / \mathrm{g}$ ), which is in close agreement with the data described in the scientific literature. ${ }^{28-30}$

Regarding the PLLA (a semicrystalline polymer) thermogram (see Fig. 3), a $T_{\text {onset }}$ of $167^{\circ} \mathrm{C}$ was produced, which is consistent with the melting temperature range of $165^{\circ} \mathrm{C}-185^{\circ} \mathrm{C}$ described in the literature. ${ }^{31}$ This same event was observed, but at a much lower intensity, in the thermograms of the SD formulations produced, viz., L3, L5, and L9. The energy absorbed (melting enthalpy) by AZT and PLLA (viz., $\Delta H_{\mathrm{AZT}}$ and $\Delta H_{\text {PLLA }}$, respectively) was calculated as the ratio of the value of integral $(\Delta U, \mathrm{~mJ})$ produced to the sample weight $(\mathrm{mg})$, for each microcalorimetric assay. The experimental melting enthalpies ( $\Delta H_{\text {observed }}$ ) together with the theoretical values for the melting enthalpies ( $\left.\Delta H_{\text {theoretical }}\right)$ calculated for the L3, L5, and L9 formulations are displayed in Table 2 . Being that the values of $\Delta H_{\text {theoretical }}(\mathrm{J} / \mathrm{g})$ was calculated by Eq. (3).

$$
\Delta H_{\text {theoretical }}=\frac{m_{\mathrm{AZTinsample}} \times \Delta H_{\mathrm{AZT}}}{m_{\text {sample }}}
$$

The experimental values for the melting enthalpies were higher than the theoretical ones for all the SD formulations 

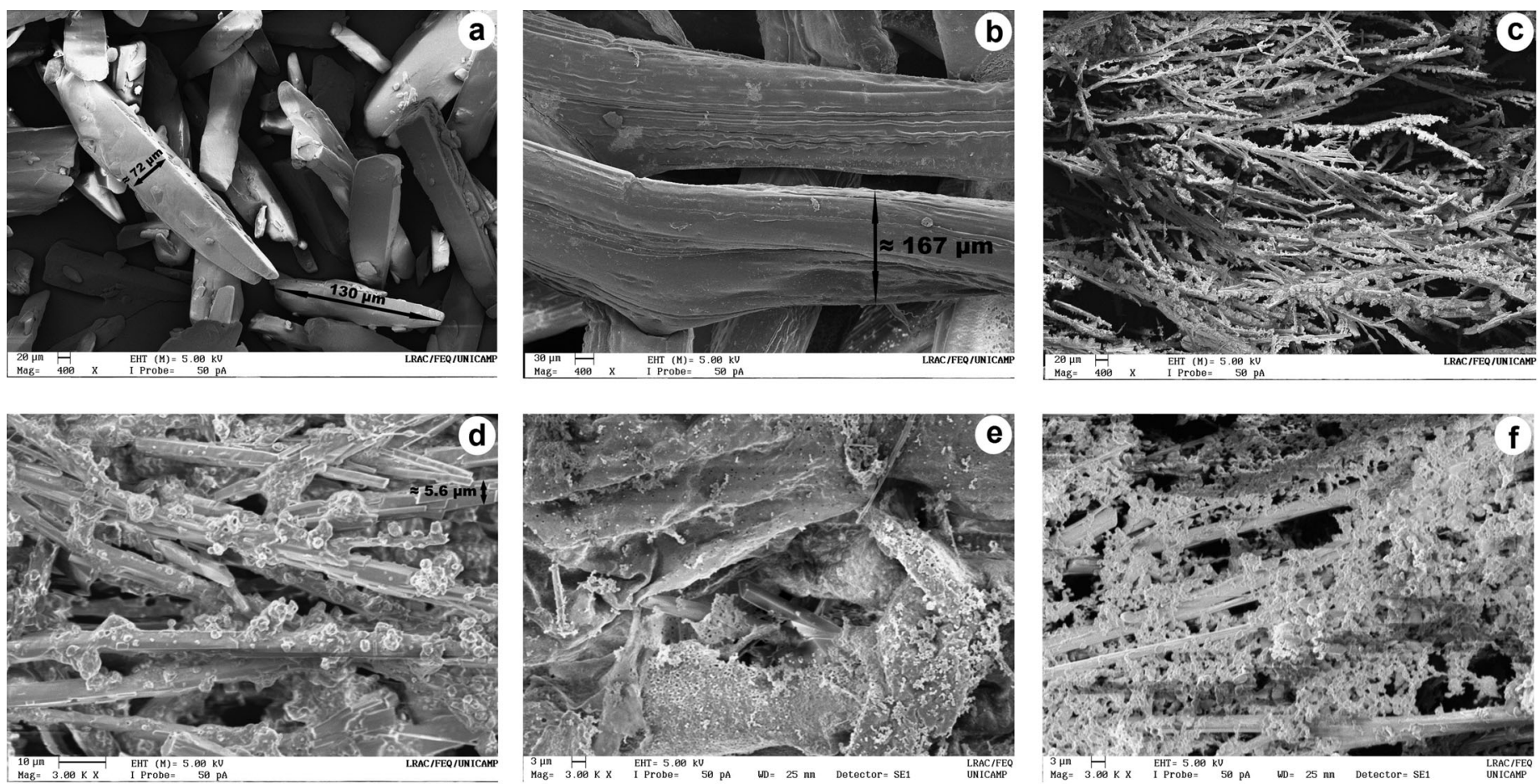

Figure 2. Scanning electron microphotographs of unprocessed AZT (a: ×400), unprocessed PLLA (b: $\times 400)$, L3 formulation (c: 400×; d: 3000×), L5 formulation (e: $3000 \times$ ), and L9 formulation (f: $3000 \times$ ).

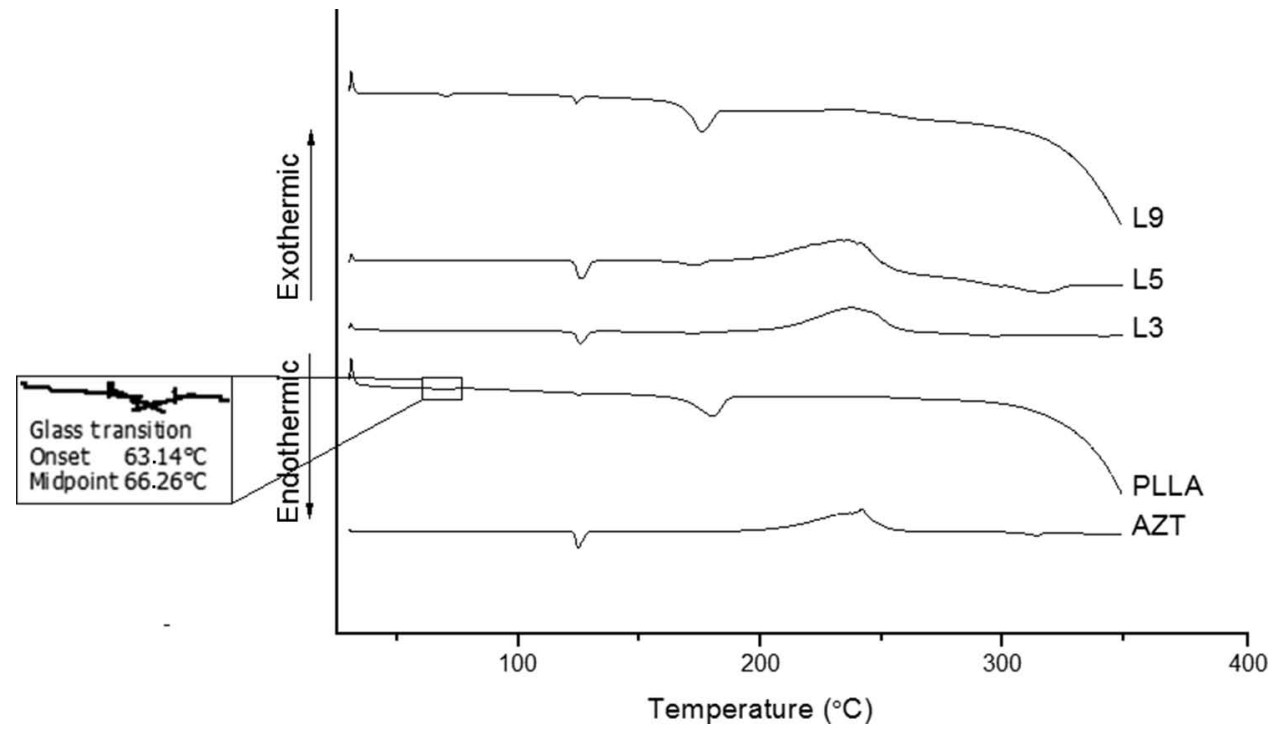

Figure 3. Normalized DSC thermograms of pure AZT, PLLA, and of L3, L5, and L9 SD systems.

Table 2. Results of both Theoretical Calculated and Observed Experimental Values for the Melting Enthalpies of all SD Formulations Assayed

\begin{tabular}{lccc}
\hline & \multicolumn{3}{c}{ Melting Enthalpy } \\
\cline { 2 - 4 } SD & $\Delta H_{\text {observed }}$ & $\Delta H_{\text {theoretical }}$ & $\Delta(\Delta H)$ \\
Formulation & $(\mathrm{J} / \mathrm{g})$ & 63.089 & 9.786 \\
\hline L3 & 72.875 & 40.432 & 51.288 \\
L5 & 91.720 & 9.453 & 11.276 \\
L9 & 22.729 & & \\
\hline
\end{tabular}

tested (see Table 2), especially in the case of SD L5 [ $\Delta H_{\text {calculated }}$ $=40.4 \mathrm{~J} / \mathrm{g} ; \Delta(\Delta H)=51.3 \mathrm{~J} / \mathrm{g}]$, showing that there was a greater demand of energy to break the intermolecular bonds than that theoretically predicted.

\section{X-ray Diffraction Analyses}

X-ray diffraction (XRD) analytical techniques are especially significant for the analysis of solid materials. The decisive advantage of XRD methods over other analytical techniques is based on the unique character of the diffraction patterns of crystalline substances, the ability to distinguish between 


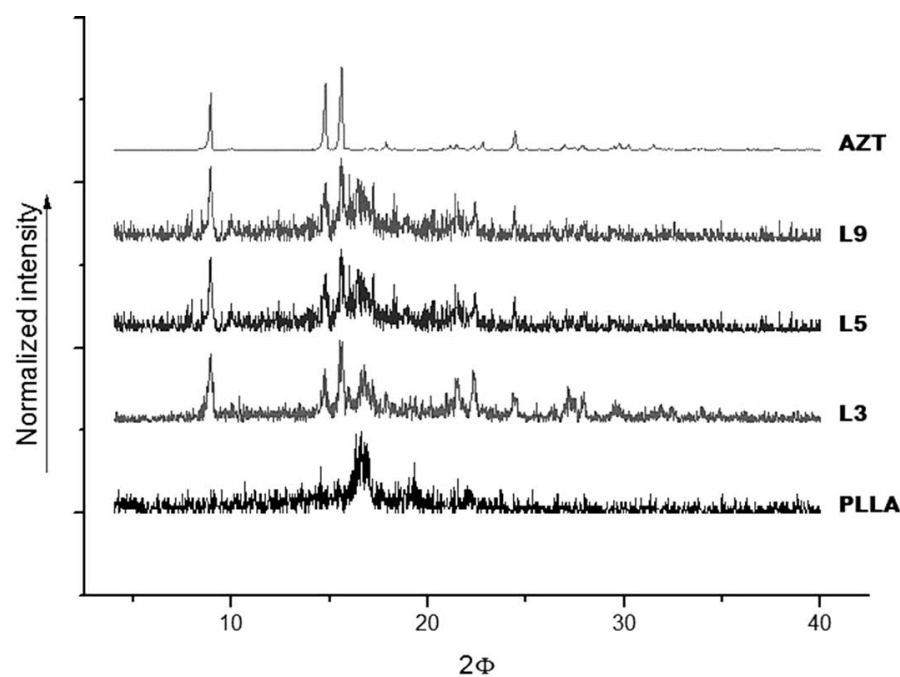

Figure 4. Normalized X-ray diffractograms for pure AZT, PLLA, and the SD systems produced (viz., L3, L5, and L9).

elements and their oxides, and the possibility to identify chemical compounds, polymorphic forms, and mixed crystals by a nondestructive examination. Hence, the crystalline state of AZT in the SD formulations was confirmed by XRD analyses. The results obtained in the XRD analysis, performed to pure AZT, PLLA, and to the three SD formulations, are displayed in Figure 4 in normalized intensity fashion.

As the intensity of the peaks in the diffraction patterns also depends on the drug concentration, a decrease in the size of peaks was expected for the SD systems encompassing lower AZT-PLLA ratios. The X-ray diffractogram of pure AZT indicates the crystalline structure of the drug, whereas the diffractograms of the SD systems clearly present the same pattern. Normalization of the intensity values in all X-ray diffractograms was made by dividing the diffractograms by the highest peak value in each diffraction pattern, thus allowing a better comparison between all X-ray diffractograms. The AZT diffractogram exhibited several peaks, indicating that it is a crystalline solid. Regarding PLLA, its diffractogram exhibited a wide background, characteristic of an amorphous compound (without fine-tuned baseline and well-defined peaks), except in the range of $16.04 \leq 2 \theta \leq 17.02$, where the small peaks produced indicate the crystalline residues of PLLA, thus allowing one to characterize this compound as a glassy amorphous polymer, ${ }^{8}$ an interpretation that is reinforced by the presence of similar small peaks in all the SD systems analyzed. The maintenance of AZT crystalline state in L3, L5, and L9 SD systems can be clearly observed in the diffractograms of those SD systems (see Fig. 4), which show sharp peaks related to AZT in the broadband referring to PLLA.

\section{Analysis by Fourier Transform Infrared Spectroscopy}

Results obtained in the FTIR analyses performed to pure AZT, PLLA, and SD systems L3, L5, and L9, are displayed in Figure 5.

As can be observed from a close inspection of Figure 5, the FTIR spectra of pure AZT and of the SD systems (L3, L5, and L9) all exhibit the characteristic stretching bands of the $\mathrm{CO}$ at $1683 \mathrm{~cm}^{-1}$ and of the azide group at $2083 \mathrm{~cm}^{-1}$. One stretching band at $1380 \mathrm{~cm}^{-1}$ can be attributed to $\mathrm{CH}_{2}$ and one stretching

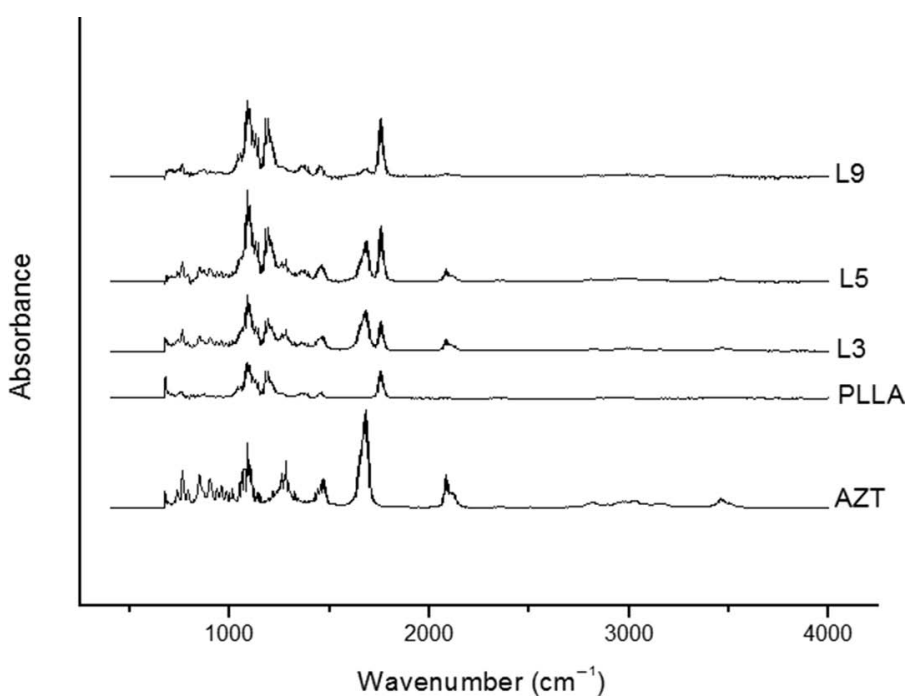

Figure 5. Fourier transform infrared spectra of pure AZT, PLLA, and the SD systems produced (viz., L3, L5, and L9).

Table 3. Drug Load and Encapsulation Efficiency of the AZT SD L3, L5, and L9

\begin{tabular}{lccccc}
\hline Theoretical & $\begin{array}{c}\text { AZT } \\
\text { AZT Loading } \\
(\%, \mathrm{w} / \mathrm{w})\end{array}$ & $\begin{array}{c}\text { AZT } \\
(\mu \mathrm{g} / \mathrm{mL})\end{array}$ & $\begin{array}{c}\text { FZT } \\
(\mu \mathrm{g} / \mathrm{mL})\end{array}$ & $\begin{array}{c}\text { Loading } \\
(\%)\end{array}$ & $\begin{array}{c}\text { AZT } \\
\text { Efficiency } \\
(\%)\end{array}$ \\
\hline L3 & 33 & 6.97 & 4.41 & 13.1 & 39.8 \\
L5 & 50 & 13.42 & 1.07 & 23.0 & 46.0 \\
L9 & 67 & 5.12 & 0.05 & 7.0 & 10.4 \\
\hline
\end{tabular}

at $1281 \mathrm{~cm}^{-1}$ can be assigned to $\mathrm{C}-\mathrm{O}-\mathrm{C}$ and $\mathrm{C}-\mathrm{OH}$ groupings. The intervals of the stretching bands produced in the research effort described herein were similar to those obtained by Araújo et al. $^{30}$ and $\mathrm{Fu}$ et al., ${ }^{32}$ and indicate the stable nature of AZT during the production process of the SD systems.

It can be seen in Figure 5 that the L3, L5, and L9 samples show the same bands as the PLLA: stretching of the CO at $1750-1760 \mathrm{~cm}^{-1}$ and peaks in the $1080-1100 \mathrm{~cm}^{-1}$ region corresponding to $\mathrm{C}-\mathrm{O}$ stretching. The peaks at 1380 and $1450 \mathrm{~cm}^{-1}$ were assigned to $\mathrm{CH}_{3}$ and $\mathrm{CH}$ bending. More detailed frequencies and their corresponding vibrational modes can be found in the work of Garlotta. ${ }^{33}$ The crystalline and amorphous phases were present in the PLLA and supercritical processed samples. This fact was corroborated by XRD.

\section{AZT Loading in L3, L5, and L9 Systems}

Results obtained for the AZT loading efficiency following determinations of AZT in the SD systems produced are showed in Table 3. The AZT in the samples evidenced that the drug was preserved during the SAS process. Among the samples investigated, L5 SD showed higher drug content.

\section{In Vitro Release Behavior of AZT}

The release of AZT from the L3, L5, and L9 SD systems was evaluated at $\mathrm{pH} 1.2$, as shown in Figure 6, and could be described by a modified release profile. The L3 SD system released $86 \%$ of the AZT at $24 \mathrm{~h}$, whereas L5 SD system released only approximately $76 \%$ AZT over $24 \mathrm{~h}$ and L9 SD system released almost $100 \%$ over $6 \mathrm{~h}$. The difference in release profiles among 


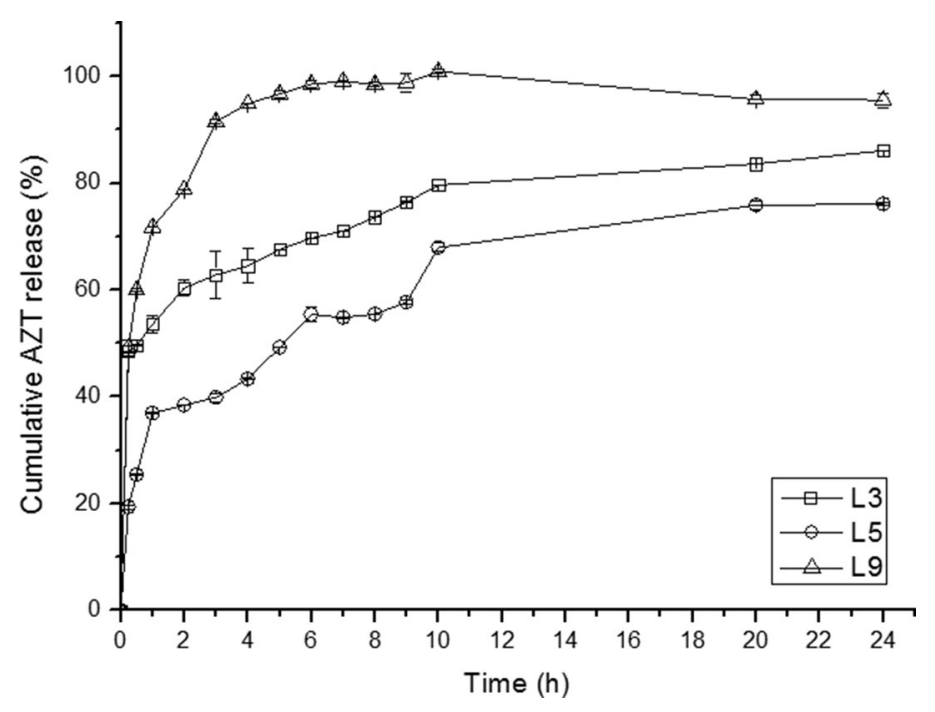

Figure 6. Release profile of AZT from the SD systems produced.

the three samples is related to the encapsulation efficiency and the degradation of PLLA. The release profile of the samples L3, L5, and L9 (Fig. 6) corresponds to the free AZT rate or adsorbed to the surface of the polymer (Table 3 ). The release of AZT from the SD systems is directly related to the stages of PLLA degradation. According to Severino et al., ${ }^{34}$ PLLA, being an aliphatic polyester, is degraded in four consecutive steps: hydration, initial degradation, subsequent degradation, and solubilization. During the PLLA hydration stage, the aqueous medium within which the polymer is submerged penetrates in the polymeric matrix, producing a relaxation of the polymer fibers and leading to a decrease of the glass transition temperature. The initial degradation of PLLA begins in the hydrated polymer via hydrolysis of the ester linkages, resulting in the cleavage of the polymer main chain, releasing lactic acid, and thus promoting a reduction of PLLA molecular weight. The hydrolysis reaction continues inside the hydrated polymer matrix in such a way that the molecular weight of the polymer continuously decreases while maintaining its integrity. During the subsequent degradation of the polymer chain, its molecular weight decreases to a point where the polymer cannot continue to maintain its integrity, and thus the polymer starts to loose mass because of solubilization in the aqueous medium. In the Biopharmaceutical Classification System, ${ }^{6} \mathrm{AZT}$ is characterized by being highly soluble and, although dispersed in the SD systems, it was fully solubilized following PLLA hydration and subsequent degradation stages.

\section{In Vitro AZT Release Kinetics}

The dissolution pattern of AZT was successfully modeled according to three models, that is, first-order, Higuchi, and Korsmeyer-Peppas kinetic models, and duly compared in order to evaluate the release kinetics. ${ }^{25}$ Results obtained are displayed in Table 4. The first-order kinetic model describes the dissolution of a drug from dosage forms such as those containing water-soluble drugs in porous matrixes. ${ }^{25}$ In this model, for each SD system, the natural logarithm of dissolved amount of AZT in the immediately preceding time was plotted versus time. The Higuchi model describes the dissolution of a drug from various types of modified-release dosage forms, such as in
Table 4. Results from the Nonlinear Fittings of the Three Kinetic Models to the Dissolved Amount of Released AZT Produced by the Different SD Systems L3, L5, and L9

\begin{tabular}{|c|c|c|c|c|c|c|c|}
\hline \multirow[b]{3}{*}{ SD System } & \multicolumn{7}{|c|}{ Kinetic Model Fitted } \\
\hline & \multicolumn{2}{|c|}{ First Order } & \multicolumn{2}{|c|}{ Higuchi } & \multicolumn{3}{|c|}{ Korsmeyer-Peppas } \\
\hline & $K_{1}$ & $r$ & $K_{\mathrm{H}}$ & $r$ & $K_{\mathrm{KP}}$ & $n$ & $r$ \\
\hline L3 & 0.031 & 0.857 & 3.966 & 0 & 39.297 & 0.139 & 0.996 \\
\hline L5 & 0.001 & 0.715 & 1.937 & 0.921 & 6.210 & 0.319 & 0.989 \\
\hline L9 & 0.022 & 0.974 & 4.035 & 0 & 38.606 & 0.146 & 0.949 \\
\hline
\end{tabular}

the case of some patches and matrix tablets with incorporated water-soluble drugs. In this model, for each SD system, the square root of time was plotted against the dissolved amount of AZT in the immediately preceding time. Korsmeyer et al. ${ }^{35}$ have developed a semiempirical simple model for describing the exponential drug release in time, which describes the release of a drug from a polymeric model system. Later, Peppas ${ }^{36}$ used the exponent release value $(n)$ to characterize different release mechanisms, and concluded that the drug transport mechanism can be classified as either a non-Fickian or Fickian diffusion process.

The L3 SD system did not respond well to the Higuchi model, and exhibited a low linearity producing an $r$ value of 0.857 for the first-order kinetic model (Table 4). However, both L3 and L5 SD systems produced a good linearity in response to the Korsmeyer-Peppas model. The $n$ value for both L3 and L5 SD systems was lower than 0.5 (Table 4 ), which was an indication that the drug diffusion through the polymer proceeded according to a Fickian diffusion mechanism. Although the L5 SD system produced a release of approximately $76 \%$ AZT during a timeframe of $24 \mathrm{~h}$, it also produced $r$ linearity for the Higuchi model, reinforcing the suggestion that the release of AZT proceeded as a diffusion mechanism based on Fick's law and, therefore, this model can be used to describe the dissolution of a drug according to a modified-release profile. ${ }^{25}$ The L9 SD system drug release kinetics displayed linearity for the first-order model, thus confirming the dissolution of AZT for release. However, for the L9 SD system, the $r$ value obtained for the Korsmeyer-Peppas model (0.949) was very similar to that obtained for the first-order model (0.974), and if we consider $n$, the Fickian diffusion is again suggested as a mechanism for the transport of AZT (Table 4). On the basis of this analysis, we can conclude that the release of AZT in the dissolution medium $(\mathrm{pH}$ 1.2 or 7.0) depends on the hydrolytic degradation of the PLLA followed by diffusion of the drug.

\section{Evaluation of Ex Vivo Intestinal Permeation}

3'-Azido-2' 3 '-dideoxythymidine intestinal permeation was evaluated using the ERIS model and the results obtained are displayed in Figure 7. To minimize the use of animals in the experimental trials, the L3 SD system was selected because of both its macroscopic characteristics (uniform particle aspect) and yield (58.7\%). Hence, the ex vivo intestinal permeation tests evaluated only the L3 (AZT-PLLA) SD system, and the data gathered from these trials were duly compared with the permeation of unprocessed AZT and drug-polymer physical mixture. For unpaired (two sample) $t$-test, the difference of the population means-for both AZT concentration in internal media $\left(\mathrm{AZT}_{\mathrm{IM}}\right)$ 


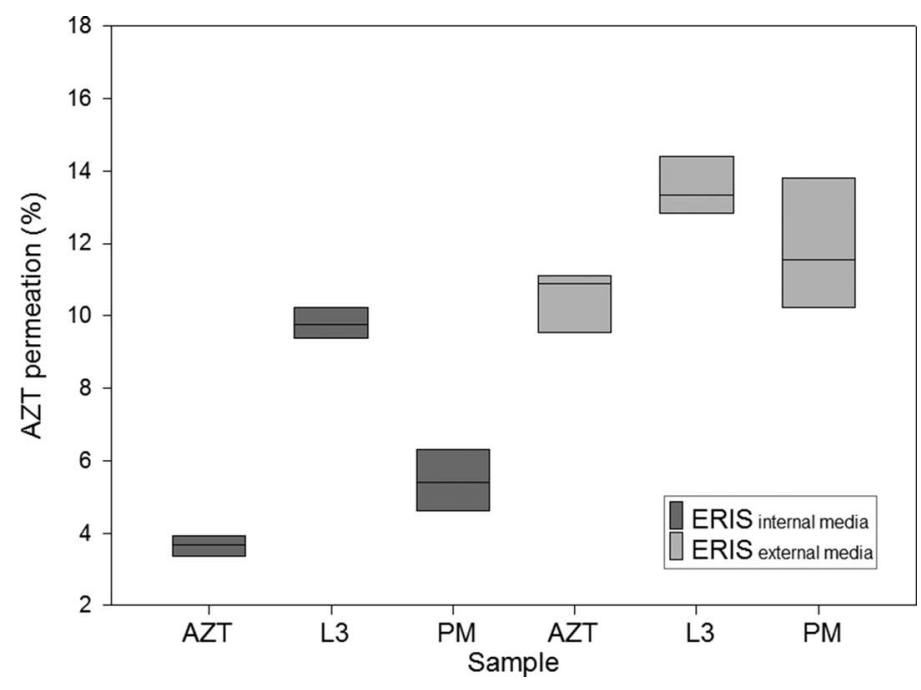

Figure 7. Quantification of AZT, for unprocessed drug (AZT), AZT from L3, and AZT from drug-polymer physical mixture (PM), in both the ERIS internal and external medium $(n=6)$, following the in vitro intestinal permeation studies.

and external media $\left(\mathrm{AZT}_{\mathrm{EM}}\right)$ for unprocessed $\mathrm{AZT}$ and $\mathrm{AZT}$ released for L3-is significantly $(p<0.05)$ different. The intestinal permeation of AZT from the L3 SD system $(9.87 \pm 0.47 \%)$ was higher than both the unprocessed AZT $(3.84 \pm 0.45 \%)$ and AZT-PLLA physical mixture $(5.40 \pm 0.93 \%)$ in the ERIS internal environment (in contact with the serous membrane) (see Fig. 7), hence suggesting that the SD system produced by using SCF processing significantly $(p<0.05)$ increased the rat intestinal absorption of AZT.

\section{DISCUSSION}

In the SAS process, $\mathrm{SC}-\mathrm{CO}_{2}$ is used as antisolvent. When the droplets come into contact with the $\mathrm{SC}-\mathrm{CO}_{2}$, a very rapid diffusion phenomenon takes place, including phase separation and solute precipitation. ${ }^{37}$ The solvent volume expansion reduces the ability to promote solvation and liquid phase supersaturation, with subsequent particle formation by drug precipitation with the carrier. ${ }^{38}$

The characteristics of the solids dispersions can be attributed to the proportion of AZT and PLLA, or to the conditions of supercritical pressure in the system environment, as the conditions of temperature and $\mathrm{CO}_{2}$ flow were kept constant for all formulations.

The AZT theoretical loading rate for the formulations L3, L5, and L9 were, respectively, 33\% (1:2), 50\% (1:1), and 67\% (2:1). However, for lower drug theoretical rates, the incorporation was higher, $40 \%$ (L3), 46\% (L5), and 10\% (L9). This can be attributed to precipitation of AZT during the SD formation, whereas the PLLA remained in solution because of the higher lipophilicity of the system, and did not coprecipitated with AZT until the solubility limit was reached. When AZT-PLLA ratio increased, the $\mathrm{SC}-\mathrm{CO}_{2}$ was saturated with PLLA gradually increasing the incorporation efficiency of AZT in SDs to more than $40 \%$, for L3 and L5 (Table 3). When AZT-PLLA ratio decreased, saturation SC- $\mathrm{CO}_{2}$ was insufficient to promote coprecipitation, decreasing the efficiency of incorporation of AZT, for L9. The interpretation of these results is in agreement with other studies. ${ }^{39,40}$ Bodmeier and McGinity ${ }^{41}$ reported that the partitioning of substances into the external phase during particles formation is a problem commonly seen with technique using immiscible phase.

The mass of components integrating the formulations that was not recovered in the SDs may have been discharged together with the solvent during its removal from the vessel of precipitation (Fig. 1). The mass transfer behavior of the fluid droplets is regarded as a critical factor in the process, influencing yield, particle morphology, and size. Dukhin et al. ${ }^{42,43}$ demonstrated the existence of droplets in transient conditions slightly above the mixture critical point, because of the existence of a dynamic interfacial tension. Consequently, the description of mass transfer from a single droplet, even under miscible conditions, appears reasonable.

In SEM, figures can observe the change in the morphology of both AZT and PLLA in the SD form and a rearrangement of the polymer chains into smaller fractions and nanoparticles of AZT onto the polymer surface.

Results obtained in this are in close agreement with those described in the literature..$^{29,44,45}$ Hence, results from both the XRD and DSC analyses of the L3, L5, and L9 SD systems strongly suggest that AZT is in crystalline form in those systems.

The aforementioned PLLA degradation stages, coupled with the data produced in the study of the AZT release kinetics (see Table 4), clearly indicates that AZT was solubilized during the PLLA hydration stage, and further released from L3, L5, and L9 SD systems by a diffusion mechanism during the initial stages of PLLA degradation.

From SEM, observations are evident that the AZT particles increased in specific surface area, which may explain the observed change in the dissolution rate. The molecular diffusion of AZT through the polymer is an efficient, simple, and reliable means to achieve controlled-release of the $\mathrm{AZT}$. The SD is a reservoir type device that utilizes this phenomenon. The main steps in the release of AZT from SDs were diffusion into the reservoir, dissolving carrier fluid between the reservoir and the polymer membrane, diffusion through the membrane, partition between membrane and eluting fluid, and finally, transport away from the device surface. ${ }^{46}$ If the reservoir contains an excess of AZT, the concentration profile at steady state can be maintained. However, after depletion of the reservoir, the concentration profile will change.

The absorption of AZT depends on its intrinsic characteristics of both solubility and permeability (ability to diffuse across the apical membrane of the lipophilic enterocytes, based on appearance of the drug in the serosal side and disappearance of the drug from the mucosal side) in the aqueous environment of the intestinal lumen. Results produced in this study are supported by the results of AZT release from the L3 SD system by a modified-release pattern of the drug. AZT is recognized as a BCS Class III drug ${ }^{6}$ with high solubility and low permeability. Hence, in the present research effort, AZT absorption from the L3 SD system was highly enhanced relative to its pure counterpart and so AZT release from the aforementioned SD system should be studied for a longer timeframe $(>10 \mathrm{~h})$ using the ERIS model to generate more extensive data comparable to the dissolution test data. 


\section{CONCLUSIONS}

The experimental results presented clearly demonstrate that it is possible to produce modified drug release systems via a SAS process for a BCS class III drug that is primarily absorbed in the stomach and in the duodenum. Results obtained show that the dissolution and absorption properties of AZT were enhanced as a consequence of PLLA degradation, and that the AZT integrated in a SD system with PLLA allowed to modify its in vitro release.

\section{ACKNOWLEDGMENTS}

The authors wish to thank Cristalia (Itapira, Brazil) for the kind supply of the reference substance used throughout the research work. Financial support from Fundação de Amparo à Pesquisa do Estado de São Paulo (2013-19300-4; 2012/013330; 2011/21219-5), Coordenação de Aperfeiçoamento de Pessoal de Nível Superior (PROSUP/CAPES) and Finep Inovação e Pesquisa (Finep, Brazil; 01.13.0286.00).

\section{REFERENCES}

1. das Neves J, Amiji MM, Bahia MF, Sarmento B. 2010. Nanotechnology-based systems for the treatment and prevention of HIV/AIDS. Adv Drug Deliv Rev 62:458-477.

2. von Briesen H, Ramge P, Kreuter J. 2000. Controlled release of antiretroviral drugs. AIDS Rev 2:31-38.

3. Ojewole E, Mackraj I, Naidoo P, Govender T. 2008. Exploring the use of novel drug delivery systems for antiretroviral drugs. Eur J Pharm Biopharm 70:697-710.

4. Mitsuya H, Weinhold KJ, Furman PA, Clair MHS, Lehrman SN, Gallo RC, Bolognesi D, Barry DW, Broder S. 1985. 3'Azido-3'deoxythymidine (BW A509U): An antiviral agent that inhibits the infectivity and cytopathic effect of human T-lymphotropic virus type III/lymphadenopathy-associated virus in vitro. Proc Natl Acad Sci USA 82:7096-7097.

5. Rachlis AR. 1990. Zidovudine (retrovir) update. Can Med Assoc J 143:1177-1185.

6. Amidon GL, Lennemas H, Shah VP, Crison JR. 1995. A theoretical basis for a biopharmaceutic drug classification: The correlation of in vitro drug product dissolution and in vivo bioavailability. Pharm Res 12:413-420.

7. Bazzo CB, Silva MAS. 2005. Estudo termo analítico de comprimidos revestidos contendo captopril através de termogravimetria (TG) e calorimetria exploratória diferencial (DSC). Braz J Pharm Sci 41:315322.

8. Piemonte V, Gironi F. 2013. Lactic acid production by hydrolysis of poly(lactic acid) in aqueous solutions: An experimental and kinetic study. J Polym Environ 21:275-279.

9. Kawashima Y, Niwa T, Handa T, Takeuchi H, Iwamoto T, Itoh K. 1989. Preparation of controlled-release microspheres of Ibuprofen with acrylic polymers by a novel quasi-emulsion solvent diffusion method. J Pharm Sci 78:68-72.

10. Benita S, Barkai A, Pathak YVJ. 1990. Effect of drug loading extent on the vitro release kinetic behaviour of nifedipine from polyacrylate microspheres. J Control Release 12:213-222.

11. Jung J, Perrut M. 2001. Particle design using supercritical fluids: Literature and patent survey. J Supercrit Fluids 20:179-219.

12. Lee S, Nam K, Kim MS, Jun SW, Park JS, Woo JS, Hwang SJ. 2005. Preparation and characterization of solid dispersion of itraconazole by using aerosol solvent extraction system for improvement in drug solubility and bioavailability. Arch Pharm Res 28:866-874.

13. Joseph NM, Sharma PK. 2008. Nanoparticle: Drug delivery system for cancer therapy. Asian J Pharm 2:139-140.
14. Zhang S, UludaĞ H. 2009. Nanoparticulate systems for growth factor delivery. Pharm Res 26:1561-1580.

15. De Zordi N, Moneghin M, Kikic I, Grassi M, Castillo AADR, Solinas D, Bolger MB. 2012. Applications of supercritical fluids to enhance the dissolution behaviors of furosemide by generation of microparticles and solid dispersions. Eur J Pharm Biopharm 81:131-141.

16. Chan H-K, Kwok PCL. 2011. Production methods for nanodrug particles using the bottom-up approach. Adv Drug Deliv Rev 63:406416.

17. Martín Á, Cocero MJ. 2004. Numerical modelling of jet hydrodynamics, mass transfer and crystallization kinetics in the supercritical antisolvent (SAS) process. J Supercrit Fluids 32:203-219.

18. Perrut M. 2000. Supercritical fluid applications: Industrial developments and economic issue. Ind Eng Chem Res 39:4531-4535.

19. Shariati A, Peters CJ. 2003. Recent developments in particle design using supercritical fluids. Curr Opin Solid State Mater Sci 7:371-383. 20. Reverchon E, Adami R. 2006. Nanomaterials and supercritical fluids. J Supercrit Fluids 37:1-22.

21. Reverchon E, Torino E, Dowy S, Brauer A, Leipertz A. 2010. Interactions of phase equilibria, jet fluid dynamics and mass transfer durins supercritical antisolvent micronization. Chem Eng J 156:446-458.

22. Sekiguchi K, Obi N. 1961. Studies on absorption of eutectic mixture. I. A comparison of the behavior of eutectic mixture of sulfathiazole and that of ordinary sulfathiazole in man. Chem Pharm Bull 9:866-872.

23. Chaud MV, Tamascia P, Lima AC, Paganelli MO, Gremião MPD, Freitas O. 2010. Solid dispersion with hydrogenated castor oil increase solubility, dissolution rate and intestinal absorption of praziquantel. Braz J Pharm Sci 46:474-481.

24. Chiou WL, Riegelman S. 1969. Preparation and dissolution characteristics of several fast-release solid dispersions of griseofulvin. J Pharm Sci 58:1505-1510.

25. Costa P, Lobo JMS. 2001. Modeling and comparison of dissolution profiles. Eur J Pharm Sci 13:123-133.

26. Correa EM, Vila MMDC, Oliveira JM Jr., Zaparoli RE, Granato MM, Goes AL, Moraes LC, Paula FC, Chaud MV. 2011. Assessment of solubility and intestinal absorption in vitro of Praziquantel in solid dispersions of polyethylene glycol 6000. Acta Farm Bonaer 30:1910 1915.

27. Ferrari PC, Souza FM, Giorgetti L, Oliveira GF, Chaud MV, Ferraz HG, Evangelista RC. 2012. In vitro drug permeation from chitosan pellets. Carbohydr Polym 87:2526-2531.

28. Yoshida VMH, de Oliveira Junior JM, Gonçalves MM, Vila MMDC, Chaud MV. 2011. Development and evaluation of a floating multiparticulate gastroretentive system for modified release of AZT. AAPS PharmSciTech 12:658-664.

29. Yoshida VMH, Granato E, Gremião MPD, Balcão VM, Vila MMDC, de Oliveira Junior JM, Severino P, Souto EB, Chaud MV. 2013. A novel gastroretentive floating system for zidovudine, based on calciumsilicate beads. Afr J Pharm Pharmacol 7:2937-2946.

30. Araújo AA, Storpirtis S, Mercuri LP, Carvalho FM, dos Santos Filho M, Matos JR. 2003. Thermal analysis of the antiretroviral zidovudine (AZT) and evaluation of the compatibility with excipients used in solid dosage forms. Int J Pharm 260:303-314.

31. Chu CC, Campbell ND. 1982. Scanning electron microscopic study of the hydrolytic degradation of poly(glycolic acid) suture. J Biomed Mater Res 16:417-430.

32. Fu K, Griebenow K, Hseih L, Klibanov VM, Langer R. 1999. FTIR characterization of the secondary structure of proteins encapsulated within PLGA microspheres. J Control Release 58:357-366.

33. Garlotta D. 2001. A literature review of poly(lactic acid). J Polym Environ 9:63-84.

34. Severino P, Santana MHA, Malmonge SM, Souto EB. 2011. Polímeros usados como sistemas de transporte de princípios ativos. Polímeros 21:361-368.

35. Korsmeyer R, Gurny R, Peppas N. 1983. Mechanisms of solute release from porous hydrophilic polymers. Int J Pharm 15:25-35.

36. Peppas NA. 1985. Analysis of Fickian and non-Fickian drug release from polymers. Pharm Acta Helv 60:110-111. 
37. Chong GH, Yunus R, Abdullah N, Choong TSY, Spotar S. 2009. Coating and encapsulation of nanoparticles using supercritical antisolvent. Am J Appl Sci 6:1352-1358.

38. Montes A, Tenorio A, Gordillo MD, Pereyra CM, de la Ossa EJM. 2011. Supercritical antisolvent precipitation of ampicillin in complete miscibility conditions. Ind Eng Chem Res 50:23432347.

39. Bodmeier R, Wang H, Dixon DJ, Mawson S, Johnston KP. 1995. Polymeric microspheres prepared by spraying into compressed carbondioxide. Pharm Res 12:1211-1217.

40. Patomchaiviwat V, Paeratakul O, Kulvanich P. 2008. Formation of inhalable rifampicin-poly(l-lactide) microparticles by supercritical anti-solvent process. AAPS PharmSciTech 9:1119_ 1129.

41. Bodmeier R, McGinity JW. 1987. The preparation and evaluation of drug-containing poly(dl-lactide) microspheres formed by the solvent evaporation method. Pharm Res 4:465-471.
42. Dukhin SS, Zhu C, Dave R, Pfeffer R, Luo JJ, Chavez F, Shen Y. 2003. Dynamic interfacial tension near critical point of a solventantisolvent mixture and laminar jet stabilization. Colloid Surf A 229:181-199.

43. Dukhin SS, Shen Y, Dave R, Pfeffer R. 2005. Droplet mass transfer, intradroplet nucleation and submicron particle production in twophase flow of solvent-supercritical antisolvent emulsion. Colloid Surf A: Physicochem Eng Asp 261:163-176.

44. Raviolo MA, Briñón MC. 2011. Preformulation studies of zidovudine derivatives: Acid dissociation constants, differential scanning calorimetry, thermogravimetry, X-ray powder diffractometry and aqueous stability studies. Sci Pharm 79:479-491.

45. Gupta RB, Chattopadhyay P. 2003. Method of forming nanoparticles and microparticles of controllable size using supercritical fluids with enhanced mass transfer. Patent US 6620351 B2.

46. Fan LT, Singh SK. 1989. Controlled release: A quantitative treatment. New York: Springer-Verlag, pp 233. 